\title{
Editorial Expression of Concern to: The fractional wavelet transform associated with the second kind of fractional Hankel transform
}

\author{
Akhilesh Prasad $^{1} \cdot$ Kanailal Mahato ${ }^{2}$ \\ Published online: 18 August 2021 \\ (C) Forum D'Analystes, Chennai 2021
}

\section{Editorial Expression of Concern to: The Journal of Analysis (2018) 26:245-257} https://doi.org/10.1007/s41478-018-0138-x

The Editor-in-Chief is issuing an Expression of Concern for this Article. The authors have notified the journal of the following potential errors:

Error 1: The kernel of the fractional Hankel transformation defined in the Eq. (1.2) which must be linear with respect to the parameter $\theta: R \propto 0 K \theta 1(t, z) K \theta 2$ $(z, \omega) d z=K \theta 1+\theta 2(t, \omega)$. In the study, the above relation doesn't hold.

Error 2: The relation defined in Eq. (1.7) (i.e. translation) is not convergent. So, the results obtained in Lemma 1.2 is not correct.

Error 3: The main goal of the paper is to study about the wavelet transformation. The wavelet transformation is defined by exploiting the term" translation". As the representation of translation is not convergent, so the wavelet transformation is also not convergent.

The authors requested a retraction, but post-publication peer-review found that it was not possible to prove or disprove the non-linearity of the operator that they have used in the paper; in line with COPE guidelines, the Editor does not consider there to be sufficient evidence the article fundamentally flawed to warrant retraction but would like to advise readers to interpret the related results with caution.

The original article can be found online at https://doi.org/10.1007/s41478-018-0138-x.

Kanailal Mahato

kanailalmahato@gmail.com; kanailalmahato@bhu.ac.in

Akhilesh Prasad

apr_bhu@yahoo.com

1 Department of Applied Mathematics, Indian Institute of Technology (Indian School of Mines), Dhanbad 826004, India

2 Department of Mathematics, Institute of Science, Banaras Hindu University, Varanasi 221005, India 
The authors have informed the journal that Akhilesh Prasad was included in error and did not consent to be an author on the manuscript.

Akhilesh Prasad and Kanailal Mahato have not responded to any correspondence from the Publisher about this Editorial Expression of Concern.

Publisher's Note Springer Nature remains neutral with regard to jurisdictional claims in published maps and institutional affiliations. 